\title{
Kinds, Distribution, and Pathogenicity of Pythium Species Isolated from Soils of Kyushu Island in Japan*
}

\author{
Tsuneo WATANABE**
}

\begin{abstract}
In assaying soil samples from 25 locations (four nine samples/Prefecture) in Kyushu Island for Pythium species by a trapping isolation method with cucumber and lupine seeds as baiting substrates, one $\sim$ seven taxa/sample were isolated from 24 samples, but no Pythium was isolated from one sample. A total of 433 isolates were identified into 16 taxa including $\mathrm{H}-\mathrm{Zs}$, a provisional Pythium group with zoospore formation from hypha-like sporangia, but without sexual organs. Among these fungi, 201 isolates of $P$. sylvaticum obtained from 22 locations were the most dominant, followed by $P$. ultimum and $P$. aphanidermatum. In inoculation experiments of 36 isolates of 16 taxa by the soil-over-agar culture inoculation method, 10 isolates including three each of $P$. sylvaticum and $P$. ultimum caused less than $60 \%$ of cucumber seeds unemerged, and after emergence, nine including two each of $P$. aphanidermatum, $P$. sylvaticum, and $P$. ultimum caused more than $40 \%$ of the seedlings collapsed or diseased. Emergence rates of komatsuna seeds appeared not to be influenced by any Pythium species tested, but after emergence, four isolates of $P$. sylvaticum, three of $P$. ultimum and one of $P$. irregulare caused more than $40 \%$ of the seedlings collapsed or diseased. Others were mildly pathogenic or nonpathogenic to both seedlings, but damage rates were below $30 \%$.
\end{abstract}

(Received September 12, 1988)

Key words: Pythium spp., kinds, distribution, taxon, Kyushu.

\section{INTRODUCTION}

Soilborne diseases of various plants due to Pythium and other pathogens have been serious recently, and therefore, ecology and distribution of these fungi have been studied in relation to etiological studies ${ }^{1,4-8,11,13-17,19)}$ or just for scientific interest ${ }^{3)}$. Kinds, and distribution of Pythium species in some Japanese soils have been reported, including piedmont natural forest at Mt. Fuji $^{16)}$, and Shikoku Island ${ }^{19)}$, and Pythium is present in almost all Japanese soils ${ }^{17}$.

This is an additional report on kinds and distribution of Pythium species isolated from soils of Kyushu Island in relation to the previous works ${ }^{13-20)}$. Pathogenicity of 36 isolates to cucumber and komatsuna seeds and seedlings was also tested

\section{MATERIALS AND METHODS}

Samples and sampling. Soil samples from 25 different locations of Kyushu Island (nine, seven, five, and four locations from Fukuoka, Kumamoto, Miyazaki and Kagoshima Pref., respectively) with various habitats, and soil $\mathrm{pH}$ values ranged from 5.6 to 7.2 were collected in August, 1980 (Table 1). The respective soil samples consisted of five random cores from the

* Part of this work was conducted at National Institute of Agricultural Sciences (presently National Institute of Agroenvironmental Sciences), Tsukuba, Japan.

** Forestry and Forest Products Research Institute, P.O. Box 16, Tsukuba Science City, Ibaraki 305, Japan 農林水産省森林総合研究所 
top $15-\mathrm{cm}$ surface soil were mixed thoroughly in polyethylene bags. These samples were usually assayed within 7 days after collection.

Isolations. Pythium was isolated by trapping methods using seeds of cucumber (Cucumis sativus L., cv. Tokiwa-jibai Kairyou) and lupine (Lupinus albus L., cv. Shirobana) as trapping substrates following the previous works ${ }^{13,17}$.

Ten seeds each embedded in each $20 \sim 50 \mathrm{~g}$ soil sample in a 9-cm petri dish incubated at $26 \mathrm{C}$ for $1 \sim 2$ days or $10 \mathrm{C}$ for $5 \sim 10$ days, respectively, were removed from soil, washed under running tap water for $1 \mathrm{hr}$, air-dried, and placed on water agar (WA, 2 seeds/plate). After a further incubation at $28 \mathrm{C}$ for $1 \sim 2$ days, pure isolates of Pythium spp. grown out of cucumber seeds on WA were obtained by single hyphal tippings.

Cultures and identifications. The fungi were cultured on various media including Difco corn meal agar for mycological study. Several soaking solutions including Petri salt solution were used to study asexual reproduction. All of fungi were identified following the descriptions by the previous workers ${ }^{9,10)}$.

Inoculations. Pathogenicty of Pythium isolates to seedlings of cucumber (cv. Shintokiwa), and komatsuna (Brassica campestris L., rapifera group, cv. Misugi) was tested in the soil-over-agar culture inoculation method ${ }^{18)}$, in which $5 \sim 10$ seeds were sown in a $50 \mathrm{ml}$ soil-overagar culture (usually 7-day-old PDA culture) in a 9-cm plastic petri dish after watering $(12 \mathrm{ml} /$ plate), and kept moist usually by placing in large petri dishes $(17.5 \mathrm{~cm}$ in diam, $18 \mathrm{~cm}$ high) one by one. The seeds sown in uninoculated soils on sterile PDA in plastic petri dishes served as controls.

Soils used for these experiments were collected from the forest nursery at the Forestry and Forest Products Research Institute at Tsukuba, Ibaraki, air-dried, and sieved through 18-mesh screen and stored in a $18 \mathrm{~cm}$ large petri dish for more than 15 days before use.

Inoculation experiments were conducted once for cucumber and komatsuna in continuous cultures in the same infested soils with two replications of a total of 10 20 plants/isolate in a growth chamber regulated at $25 \mathrm{C}$ for $12 \mathrm{hr}$ of light and $19 \mathrm{C}$ for $12 \mathrm{hr}$ of darkness.

Pathogenicity was evaluated by rates of emergence, and damaged (collapsed or diseased) seedlings/total number of seeds tested 7 days after sowing.

\section{RESULTS AND DISCUSSION}

\section{Isolations, distribution and notes of Pythium isolates}

A total of 433 Pythium isolates obtained from soils from 25 locations in Kyushu Island included 234 and 199 isolates trapped by cucumber and lupine seeds, respectively. By cucumber and lupine seeds, 13 species each were isolated, totalling at least 16 taxa including H-Zs (a provisional Pythium group with zoospore formation from hypha-like sporangia, but without sexual organ) ${ }^{19)}$ (Figs. 9 11) and two unidentified isolates. Pythium species in Kyushu Island with numbers of samples yielding each taxon, and of the isolates obtained in each taxon are listed, together with representative isolates and their origin in Table 2. Kinds of Pythium species and number of isolates obtained from each sample (location) are tabulated in Table 3.

Of the 16 taxa, 11 were commonly isolated by both seeds. However, one isolate each of $P$. elongatum Matthews (Figs. $1 \sim 3$ ) and $P$. dissotocum Drechsler were isolated only by cucumber seeds, and two of $P$. inflatum Matthews (Figs. $4 \sim 8$ ) and one of $P$. myriotylum Drechsler, only by lupine seeds.

Influence of temperatures for isolation of Pythium species was previously discussed ${ }^{13)}$. However, of the 205 isolates including 115 trapped by cucumber seeds and 90 by lupine seeds at $26 \mathrm{C}$ and 228 including 119 by cucumber seeds and 109 by lupine seeds at $10 \mathrm{C}, 11$ taxa were obtained at $26 \mathrm{C}$ by both seeds, whereas eight or six obtained at $10 \mathrm{C}$ by cucumber and lupine seeds, respectively. Especially, five Pythium species, i.e., P. aphanidermatum (Edson) Fitz., $P$. elongatum, $P$. hydnosporum (Mont.) Schroeter, $P$. inflatum, and $P$. torulosum Coker \& Patterson 
were isolated only at $26 \mathrm{C}$, but not at $10 \mathrm{C}$. The rest of at least 11 taxa were isolated at either temperatures. In addition, 11 isolates of $P$. graminicola Subramaniam and 12 of $P$. splendens Braun were isolated at $26 \mathrm{C}$, but one isolate each of both species at $10 \mathrm{C}$, whereas 64 isolates of P. ultimum Trow were isolated at $10 \mathrm{C}$, but only 27 isolates at $26 \mathrm{C}$.

The respective of 24 samples yielded one to seven species, but no Pythium was isolated from one sample, i.e., cucumber field soil at Munakata with sample no. 64-s in Fukuoka Pref., although Phytophthora sp. was trapped (Table 3). A total of 66 isolates with lobate sporangia included 51 of $P$. aphanidermatum collected in 13 locations, 12 of $P$. graminicola from three locations, two of $P$. torulosum from one location and an unidentified isolate (Isolate 80-379). Both $P$. aphanidermatum and $P$. torulosum discharged zoospores easily, but $P$. graminicola and an unidentified isolate were not easy for discharging zoospores.

Of 13 isolates with zoospore formation from hypha-like sporangia, one isolate was $P$. dissotocum, and 12 from two locations were H-Zs. This H-Zs may be or close to P. flevoense Van der Plaats-Niterink, a heterothallic species with hypha-like sporangia ${ }^{10}$.

Three isolates collected in cucumber field at Fukuoka Pref. formed sphaerosporangia with zoospore discharge and they were all identified as $P$. vexans de Bary. Another unidentified isolate (Isolate 80-269) with sphaerosporangia with zoospore discharge is close to $P$. intermedium de Bary because of formation of short chains of basipetally-developed sporangia, but different from it because of nondesiduous nature of sporangia.

Of a total of 41 isolates with echinulate oogonia and spherical hyphal swellings (conidia), five isclates were tentatively identified as $P$. hydnosporum, although one isolate from cabbage field at Kagoshima Pref., was slightly different from four others with rather inconspicuous echinulation and half-sized globoid structure. Nine isolates from four locations were $P$. irregulare Buisman, and 27 from eight locations were P. spinosum Sawada.

Of 304 isolates forming spherical hyphal swellings without zoospore formation, 13 isolates were $P$. splendens with terminal large hyphal swellings (usually more than $30 \mu \mathrm{m}$ in diam) and rarely oogonia with diclinous $1 \sim 2$ antheridia and aplerotic oospores, although conidia of Isolate 80-212 were more frequently intercalary than terminal. A total of 91 isolates from 17 locations were $P$. ultimum and included three isolates (Isolates $80-289 ;-366 ;-615$ ) with predominantly hypogynous antheridia.

Table 1. Soil samples obtained from 25 different locations with various habitats and soil $\mathrm{pH}$ values in Kyushu Island

\begin{tabular}{|c|c|c|c|c|c|c|c|}
\hline $\begin{array}{c}\text { Sample } \\
\text { No. }\end{array}$ & Location & Habitat & $\mathrm{pH}$ & $\begin{array}{c}\text { Sample } \\
\text { No. }\end{array}$ & Location & Habitat & $\mathrm{pH}$ \\
\hline \multicolumn{4}{|c|}{ Fukuoka Pref. } & \multicolumn{4}{|c|}{ Kumamoto Pref. } \\
\hline 53 & Munakata & Cabbage & 5.6 & 66 & Kumamoto & Corn & 6.9 \\
\hline 55 & Munakata & Watermelon & 6.5 & 68 & Ueki & Eggplant & 7.2 \\
\hline 56 & Munakata & Watermelon & 6.4 & 70 & Ueki & Cucumber & 6.6 \\
\hline 57 & Munakata & Watermelon & 6.2 & 73 & Ueki & Cucumber & 5.8 \\
\hline 59 & Munakata & Spinach & 6.7 & 74 & Ueki & Cabbage & 6.2 \\
\hline 62 & Munakata & Cucumber & 7.1 & 76 & Ueki & Cucumber & 6.7 \\
\hline 63 & Munakata & Watermelon & 6.7 & 77 & Ueki & Cabbage & 6.8 \\
\hline 64-s & Munakata & Cucumber & 5.8 & & & & \\
\hline 65 & Munakata & Cucumber & 7.1 & & & & \\
\hline \multicolumn{4}{|c|}{ Miyazaki Pref. } & \multicolumn{4}{|c|}{ Kagoshima Pref. } \\
\hline 78 & Miyakonojyo & Taro & 6.0 & 81 & Sueyoshi & Cabbage & 6.7 \\
\hline 79 & Miyakonojyo & Peanut & 6.6 & 82 & Kouyama & Sweet potato & 6.8 \\
\hline 80 & Miyakonojyo & Soybean & 6.3 & 83 & Kaimon & Watermelon & 5.8 \\
\hline M-1 & Kiyotake & Cucumber & 6.0 & 84 & Kagoshima & Sweet potato & 6.9 \\
\hline M-2 & Kiyotake & Cucumber & 6.8 & & & & \\
\hline
\end{tabular}


Of 201 isolates with spherical hyphal swellings (conidia) identified as $P$. sylvaticum Campbell $\&$ Hendrix $^{2)}$ in this study, 33 isolates occasionally formed sexual organs with aplerotic oospores and $2 \sim 3$ or more diclinous antheridia per oogonium (homothallic isolates), but others never formed sexual organs (heterothallic isolates) ${ }^{2,4)}$. Of the 33 homothallic isolates, 24 (nearly $73 \%$ ) were isolated at $10 \mathrm{C}$.

Pythium sylvaticum occurred most commonly and it was isolated from 22 locations. Pythium aphanidermatum and $P$. ultimum were isolated from 13 and 17 locations, $P$. spinosum and $P$. splendens from eight and six locations, respectively, but others were isolated from less than five locations (Tables 2 and 3).

At least 11 taxa out of 16 obtained in this study were recorded in the previous report from Shikoku Island ${ }^{19)}$, but four taxa, i.e., P. elongatum, P. inflatum, P. splendens and P. myriotylum were not recorded. However, most of these species were also recorded in soils of different parts of Japan ${ }^{13,17)}$ and other countries ${ }^{3,5,11)}$.

\section{Pathogenicity}

Although pathogenicity of Pythium species isolated from soils was demonstrated in England ${ }^{12)}$

Table 2. List of Pythium species isolated from soils of Kyushu Island, and representative isolates

\begin{tabular}{|c|c|c|c|}
\hline Pythium species & $\begin{array}{l}\text { Sample } \\
\text { yielding } \\
\text { No. }\end{array}$ & $\begin{array}{l}\text { Isolates } \\
\text { No. }\end{array}$ & $\begin{array}{l}\text { Representative isolates } \\
\text { and their origin }\end{array}$ \\
\hline P. aphanidermatum (Edson) Fltz. & 13 & 51 & $\begin{array}{l}80-140 \text { (Sample 53) } \\
80-265 \text { (Sample M-2) }\end{array}$ \\
\hline P. dissotocum Drechsler & 1 & 1 & 80-571 (Sample 83) \\
\hline P. graminicola Subramaniam & 3 & 12 & $\begin{array}{l}80-214(\text { Sample } 80)=\text { ATCC64150 } \\
80-299(\text { Sample 66) } \\
80-302(\text { Sample 66) }=\text { ATCC64151 }\end{array}$ \\
\hline$P$. elongatum Matthews & 1 & 1 & 80-222 (Sample 80) \\
\hline P. hydnosporum (Mont.) Schroeter & 4 & 5 & $\begin{array}{l}\text { 80-228 (Sample } 81) \\
\text { 80-266 (Sample M-2) } \\
\text { 80-295 (Sample 66) }\end{array}$ \\
\hline P. inflatum Matthews & 1 & 2 & $80-150 ;-152$ (Sample 55) \\
\hline$P$. irregulare Buisman & 4 & 9 & $\begin{array}{l}80-243 \text { (Sample 83) } \\
80-348 \text { (Sample 76) }\end{array}$ \\
\hline P. myriotylum Drechsler & 1 & 1 & 80-248 (Sample 84) \\
\hline$P$. spinosum Sawada & 8 & 27 & 80-203 (Sample 78) \\
\hline P. splendens Braun & 6 & 13 & $\begin{array}{l}\text { 80-212 (Sample 79) } \\
80-311 \text { (Sample 68) }\end{array}$ \\
\hline P. sylvaticum Campbell \& Hendrix & 22 & 201 & $\begin{array}{l}\text { 80-164 (Sample 56) } \\
80-225 \text { (Sample } 81) \\
80-272 \text { (Sample } \mathrm{M}-1 \text { ) } \\
\text { 80-620 (Sample } 81 \text { ) }\end{array}$ \\
\hline P. torulosum Coker \& Patterson & 1 & 2 & 80-149;-155 (Sample 55) \\
\hline P. ultimum Trow & 17 & 91 & $\begin{array}{l}80-186 \text { (Sample 62) } \\
80-247 ;-255 \text { (Sample } 84) \\
80-289 \text { (Sample 65) }\end{array}$ \\
\hline$P$. vexans de Bary & 1 & 3 & 80-274 (Sample 65) \\
\hline $\mathrm{H}-\mathrm{Zs}^{\text {a) }}$ & 2 & 12 & $\begin{array}{l}\text { 80-151 (Sample 55) } \\
80-239 ;-240 ;-241 ;-570 ;-576 \\
\quad \text { (Sample 83) }\end{array}$ \\
\hline Pythium spp. & 2 & 2 & $\begin{array}{l}\text { 80-269 (Sample M-2) } \\
80-379 \text { (Sample 55) }\end{array}$ \\
\hline
\end{tabular}

a) A provisional Pythium group with zoospore formation from hypha-like sporangia, but without sexual organs. 
Table 3. Kinds of Pythium species and number of isolates obtained from soils of 25 different locations in Kyushu Island

\begin{tabular}{|c|c|c|c|c|c|c|c|c|c|c|}
\hline \multirow{2}{*}{$\begin{array}{c}\text { Sample } \\
\text { No. }\end{array}$} & \multicolumn{10}{|c|}{ Kind of Pythium ${ }^{\text {a) }}$ and no. of isolates } \\
\hline & aph & gra & hyd & irr & spi & spl & syl & ult & $\mathrm{H}-\mathrm{Zs}$ & Miscellaneous b) \\
\hline \multicolumn{11}{|c|}{ Fukuoka Pref. } \\
\hline 53 & 1 & & & & & & 12 & 8 & & \\
\hline 55 & 2 & & & & & & 3 & 5 & 1 & tor, 2 ; inf, 2 ; Pyth. sp., 1 \\
\hline 56 & 4 & & & & 1 & & 3 & 10 & & \\
\hline 57 & 2 & & & & 3 & & 10 & 5 & & \\
\hline 59 & 7 & & & & & & 9 & 4 & & \\
\hline 62 & 7 & & & & & & 4 & 10 & & \\
\hline 63 & 1 & & & & & & 11 & 5 & & \\
\hline \multicolumn{11}{|l|}{ 64-s } \\
\hline 65 & & & & & & 1 & 3 & 10 & & vex, 3 \\
\hline \multicolumn{11}{|c|}{ Kumamoto Pref. } \\
\hline 66 & 2 & 6 & 1 & & & & 4 & 10 & & \\
\hline 68 & 3 & & & 1 & 3 & 2 & 10 & & & \\
\hline 70 & 11 & & & & 1 & 1 & & 5 & & \\
\hline 73 & & & & & & & 19 & 1 & & \\
\hline 74 & & & & & & & 23 & 2 & & \\
\hline 76 & & & & 1 & & & 16 & & & \\
\hline 77 & & & & & & & 22 & 1 & & \\
\hline \multicolumn{11}{|c|}{ Miyazaki Pref. } \\
\hline 78 & & & & & 7 & & 6 & & & \\
\hline 79 & & & & & 1 & 3 & 9 & & & \\
\hline 80 & & 5 & & & 2 & 1 & 4 & & & elo, 1 \\
\hline M-1 & 1 & & 1 & & & & 12 & & & \\
\hline M-2 & 9 & & 2 & & & & 9 & 3 & & Pyth. sp., 1 \\
\hline \multicolumn{11}{|c|}{ Kagoshima Pref. } \\
\hline 81 & 1 & & 1 & & & & 10 & 1 & & \\
\hline 82 & & & & & 9 & 5 & 1 & & & \\
\hline 83 & & & & 1 & & & 1 & 3 & 11 & dis, 1 \\
\hline 84 & & 1 & & 6 & & & & 8 & & myr, 1 \\
\hline $\begin{array}{l}\text { Total } \\
\text { isolates }\end{array}$ & 51 & 12 & 5 & 9 & 27 & 13 & 201 & 91 & 12 & 12 \\
\hline
\end{tabular}

a) Abbreviated as follows: $a p h=P$. aphanidermatum, dis $=P$. dissotocum, elo $=P$. elongatum, gra $=P$. graminicola, hyd $=P$. hydnosporum, inf $=P$. inflatum, irr $=P$. irregulare, spi $=P$. spinosum, $\mathrm{spl}=P$. splendens, syl $=P$. sylvaticum, tor $=P$. torulosum, ult $=P$. ultimum, vex $=P$. vexans, $\mathrm{H}-\mathrm{Zs}=\mathrm{a}$ provisional Pythium group with zoospore formation from hypha-like sporangia, but without sexual organs.

b) Isolated only from one location (sample), and unidentified Pythium spp.

and in the United States ${ }^{4}$, only a few reports have been published on Japanese soil isolates. These reports include data on pathogenicity of two Kyushu isolates ${ }^{15}$ ) of $\boldsymbol{P}$ aphanidermatum and 23 Shikoku isolates ${ }^{20)}$ of Pythium spp. to cucumber, and/or komatsuna and Japanese black pine seedlings. To obtain further information, 36 isolates of 16 taxa from Kyushu Island were tested for their pathogenicity to komatsuna and cucumber seedlings by the soil-over-agar culture inoculation method (Table 4). Of these isolates, 10 including $P$. splendens (Isolate 80-311) and $P$. ultimum (80-247) caused emergence rates of cucumber to $0 \sim 60 \%$, and the respective test fungi were recovered $100 \%$ from each sample. Rest of 20 isolates did not influence emergence rates of cucumber seeds, but after emergence, nine isolates including P. ultimum (80-186) were most pathogenic, causing more than $40 \%$ damage rates, seven isolates including two of $P$. graminicola were mildly pathogenic, causing less than $10 \%$ damage rates, and rest of the isolates were nonpathogenic. 
Table 4. Pathogenicity a) to seedlings of cucumber (cv. Shintokiwa), and Brassica campestris L. (rapifera group), (komatsuna, cv. Misugi) of 36 Pythium isolates from soils of Shikoku Island by the soilover-agar culture inoculation method in a growth chamber

\begin{tabular}{|c|c|c|c|c|c|c|c|}
\hline \multirow[b]{2}{*}{ Fungus } & & \multicolumn{3}{|c|}{ Cucumber } & \multicolumn{3}{|c|}{ Komatsuna } \\
\hline & & $\begin{array}{c}\text { Emerg. } \\
(\% / 0)\end{array}$ & $\underset{(\%)}{\text { Damage }}$ & $\begin{array}{c}\text { Recov. } \\
(\%)\end{array}$ & $\underset{(\%)}{\text { Emerg. }}$ & $\underset{(\%)}{\text { Damage }}$ & $\underset{(\%)}{\text { Recov. }}$ \\
\hline P. aphanidermatum & $80-140$ & 90 & 70 & 100 & 100 & 10 & 0 \\
\hline P. aphanidermatum & $80-265$ & 90 & 40 & 75 & 100 & 0 & 0 \\
\hline P. dissotocum & $80-571$ & 90 & 10 & 25 & 95 & 0 & 25 \\
\hline P. elongatum & $80-222$ & 100 & 0 & 0 & 100 & 0 & 0 \\
\hline P. graminicola & $80-214$ & 100 & 10 & 25 & 90 & 0 & 0 \\
\hline P. graminicola & $80-299$ & 100 & 10 & 25 & 95 & 5 & 50 \\
\hline P. graminicola & $80-302$ & 100 & 0 & 0 & 90 & 0 & 25 \\
\hline P. hydnosporum & $80-228$ & 90 & 0 & 0 & 90 & 0 & 0 \\
\hline P. hydnosporum & $80-266$ & 100 & 0 & 0 & 95 & 0 & 0 \\
\hline P. hydnosporum & $80-295$ & 100 & 0 & 0 & 100 & 0 & 0 \\
\hline$P$. inflatum & $80-150$ & 90 & 0 & 25 & 100 & 0 & 0 \\
\hline$P$. inflatum & $80-152$ & 100 & 10 & 25 & 90 & 0 & 0 \\
\hline$P$. irregulare & $80-243$ & 60 & 40 & 100 & 95 & 40 & 75 \\
\hline$P$. irregulare & $80-348$ & 30 & 10 & 100 & 100 & 0 & 0 \\
\hline$P$. spinosum & $80-203$ & 40 & 40 & 100 & 90 & 25 & 100 \\
\hline P. splendens & $80-212$ & 80 & 70 & 100 & 95 & 5 & 25 \\
\hline P. splendens & $80-311$ & 0 & - & 100 & 95 & 10 & 50 \\
\hline P. sylvaticum & $80-164$ & 60 & 50 & 100 & 95 & 50 & 100 \\
\hline P. sylvaticum & $80-225$ & 20 & 20 & 100 & 95 & 75 & 100 \\
\hline P. sylvaticum & $80-272$ & 70 & 60 & 100 & 100 & 65 & 100 \\
\hline P. sylvaticum & $80-620$ & 30 & 30 & 75 & 85 & 65 & 100 \\
\hline P. torulosum & $80-149$ & 100 & 0 & 0 & 85 & 0 & 0 \\
\hline P. torulosum & $80-155$ & 100 & 10 & 25 & 100 & 0 & 0 \\
\hline P. ultimum & $80-186$ & 90 & 90 & 100 & 100 & 5 & 50 \\
\hline P. ultimum & $80-247$ & 0 & - & 100 & 100 & 100 & 100 \\
\hline P. ultimum & $80-255$ & 60 & 30 & 100 & 100 & 90 & 100 \\
\hline P. ultimum & $80-289$ & 50 & 40 & 100 & 95 & 40 & 100 \\
\hline$P$. vexans & $80-274$ & 80 & 0 & 75 & 90 & 0 & 0 \\
\hline $\mathrm{H}-\mathrm{Zs}$ b) & $80-151$ & 100 & 0 & 0 & 100 & 0 & 0 \\
\hline $\mathrm{H}-\mathrm{Zs}$ & $80-239$ & 100 & 0 & 0 & 95 & 0 & 25 \\
\hline $\mathrm{H}-\mathrm{Zs}$ & $80-240$ & 90 & 10 & 100 & 95 & 5 & 75 \\
\hline $\mathrm{H}-\mathrm{Zs}$ & $80-241$ & 100 & 0 & 0 & 90 & 0 & 0 \\
\hline $\mathrm{H}-\mathrm{Zs}$ & $80-570$ & 90 & 10 & 25 & 100 & 0 & 0 \\
\hline $\mathrm{H}-\mathrm{Zs}$ & $80-576$ & 100 & 0 & 0 & 100 & 0 & 25 \\
\hline Pythium sp. & $80-269$ & 90 & 0 & 0 & 85 & 0 & 0 \\
\hline Pythium sp. & $80-379$ & 100 & 0 & 25 & 90 & 0 & 0 \\
\hline Control & & 90 & 0 & 0 & 90 & 0 & 0 \\
\hline
\end{tabular}

a) Evaluated on the basis of rates of emergence and damaged (collapsed or diseased) seedlings/total number of seeds tested 7 days after sowing. Average of 10 20 plants/isolate/test. The respective test fungi were recovered by plating $1-\mathrm{cm}$ basal hypocotyl segments of four most damaged seedlings in each test.

b) Provisionally-named Pythium group, with zoospore discharge from hypha-like sporangia, but without sexual organs.

Emergence of komatsuna seeds appeared not to be influenced by any Pythium species, but after emergence, eight isolates including two of $P$. ultimum $(80-255 ;-247)$ were most pathogenic, causing more than $40 \%$ of the seedlings collapsed or diseased, seven including $P$. spinosum (80- 
283) were mildly pathogenic, causing $5 \sim 25 \%$ damage rates, and rest of isolates were nonpathogenic.

It is interesting to note that two isolates each of $\boldsymbol{P}$. aphanidermatum and $\boldsymbol{P}$. splendens were mildly pathogenic or nonpathogenic to komatsuna, but most pathogenic to cucumber seedlings. Results of these experiments were more or less identical with the previous report ${ }^{20)}$.

I wish to thank Mr. S. Tanaka, Fukuoka Prefectural Horticultural Experiment Station, Fukuoka, Mr. T. Nakayama, Kumamoto Prefectural Agricultural Experiment Station, Kumamoto, and Mr. K. Kita, Kyushu National Agricultural Experiment Station, Miyazaki for their help in collecting soil samples. Thanks are also due to Dr. T. Araki, Institute of Japan Plant Protection Association, Ibaraki for his encouragement during this study.

\section{Literature cited}

1. Campbell, W.A. and Hendrix, F.F., Jr. (1967). Plant Dis. Reptr. 51: 929-932.

2. Campbell, W.A. and Hendrix, F.F., Jr. (1967). Mycologia 59: 274-278.

3. Dick, M.W. and Ali-Shtayeh, M.S. (1986). Trans. Br. mycol. Soc. 86: 49-62.

4. Hendrix, F.F., Jr. and Campbell, W.A. (1968). Forest Science 14: 292-297.

5. Hendrix, F.F., Jr. and Campbell, W.A. (1970). Can. J. Bot. 48: 377-384.

6. Hendrix, F.F., Jr. and Campbell, W.A. (1973). Ann. Rev. Phytopath. 11: 77-98.

7. Ichitani, T. and Shinsu, T. (1980). Ann. Phytopath. Soc. Japan 46: 435-441.

8. Klemmer, H.W. and Nakano, R.Y. (1964). Plant Dis. Reptr. 48: 848-852.

9. Middleton, J.T. (1943). Mem. Torrey Bot. Club 20: 1-171.

10. Plaats-Niterink, A.J. van der (1981). Monograph of the genus Pythium. Studies in Mycology (CBS) 21: 1-242.

11. Vaartaja, O. and Bumbieris, M. (1964). Aust. J. Biol. Sci. 17: 436-445.

12. Warcup, J.H. (1952). Trans. Brit. mycol. Soc. 35: 248-262.

13. Watanabe, T. (1981). Ann. Phytopath. Soc. Japan 47: 449-456.

14. Watanabe, T. (1981). Ibid. 47: 562-565.

15. Watanabe, T. (1983). Trans. mycol. Soc. Japan 24: 15-23.

16. Watanabe, T. (1985). Ibid. 26: 41-45.

17. Watanabe, T. (1985). Plant Prot. Bull. (Taiwan, R.O.C.) 27: 211-224.

18. Watanabe, T. (1988). Trans. mycol. Soc. Japan 29: 197-203.

19. Watanabe, T. (1988). Ann. Phytopath. Soc. Japan 54: 523-528.

20. Watanabe, T., Uematsu, S. and Inoue, Y. (1988). Ibid. 54: 565-570.

\section{和 文 摘 要}

\section{渡辺恒雄 : 九州地方の土裹から分離したP Pythium 菌の種と分布扔よび病原性}

九州地方の土壤に生息する Pythium 菌の種名と分布を明らかにするため, キュウリとルーピン種子によ る捕捉法を用いて菌を分離し, 調査した。供試した 25 カ所の土㙵 (4 9/県) 中, 24 試料から 1 試料当り 1〜7種のPythium 菌が分離されたが, 福岡県の 1 試料からはまったく分離できなかった。分離した合計 433 菌株は, H-Zs（糸状胞子囊から遊走子を形成するが，生殖器官は未形成の一群）を含を 16 種に分類・同定 できた。最も一般的に分布していたのは，P. sylvaticum で，22 カ所から 201 菌株が分離された。次いで, P. ultimum と P. aphanidermatum の分布が広く，13 17 カ所から 51 91 菌株が分離された。寒天培養覆 土接種法を用いて 16 種の 36 菌株（未同定菌を含む）の接種試験を行ったところ，P. sylvaticum と $P$. ultimum の各 3 菌株を含む合計 10 菌株はキュウリの萠芽率を $60 \%$ 以下に低下させ，また，P. aphanidermatum, P. sylvaticum 扰よび P. ultimum の各 2 菌株を含む 9 菌株は萠芽後の子苗の $40 \%$ 以上を枯死または罹 病させた。コマッナでは, いずれの処理区でも $85 \%$ 以上の萌芽率を示し, 萌芽前の被害は認められなかっ た。しかし，P. sylvaticum, P. ultimum, P. irregulare など 8 菌株はいずれも萌芽後に $40 \%$ 以上の被害率を 引き起こした。他の供試菌の病原性は，かなり強いものからまったく認められないものまで，さまざまであ った。 


\section{Explanation of plate}

\section{Plate I}

Figs. 1 11. Morphology of Pythium spp. from soils of Kyushu Island.

$1 \sim 3$. P. elongatum (Isolate 80-222)

Vesicle formation from long exit tubes or hypha-like sporangia $(1,2)$ and irregular $(1)$, globose (2) and elliptical $(1,3)$ hyphal swellings.

4 8. P. inflatum (Isolate 80-150)

Lobate sporangia $(4,5)$, oogonia with typical diclinous antheridia $(6,7)$, and a plerotic oospore (8).

9 11. H-Zs (Isolate 80-570)

Vesicle formation $(9,10)$ from hypha-like sporangia, and filamentous dendroid hyphae (11).

Scale bar (Fig. 3) $=20 \mu \mathrm{m}$ in Figs. $1 \sim 6,9,11$; bar $=10 \mu \mathrm{m}$ in Figs. 7, 8, 10. 


\section{Plate I}

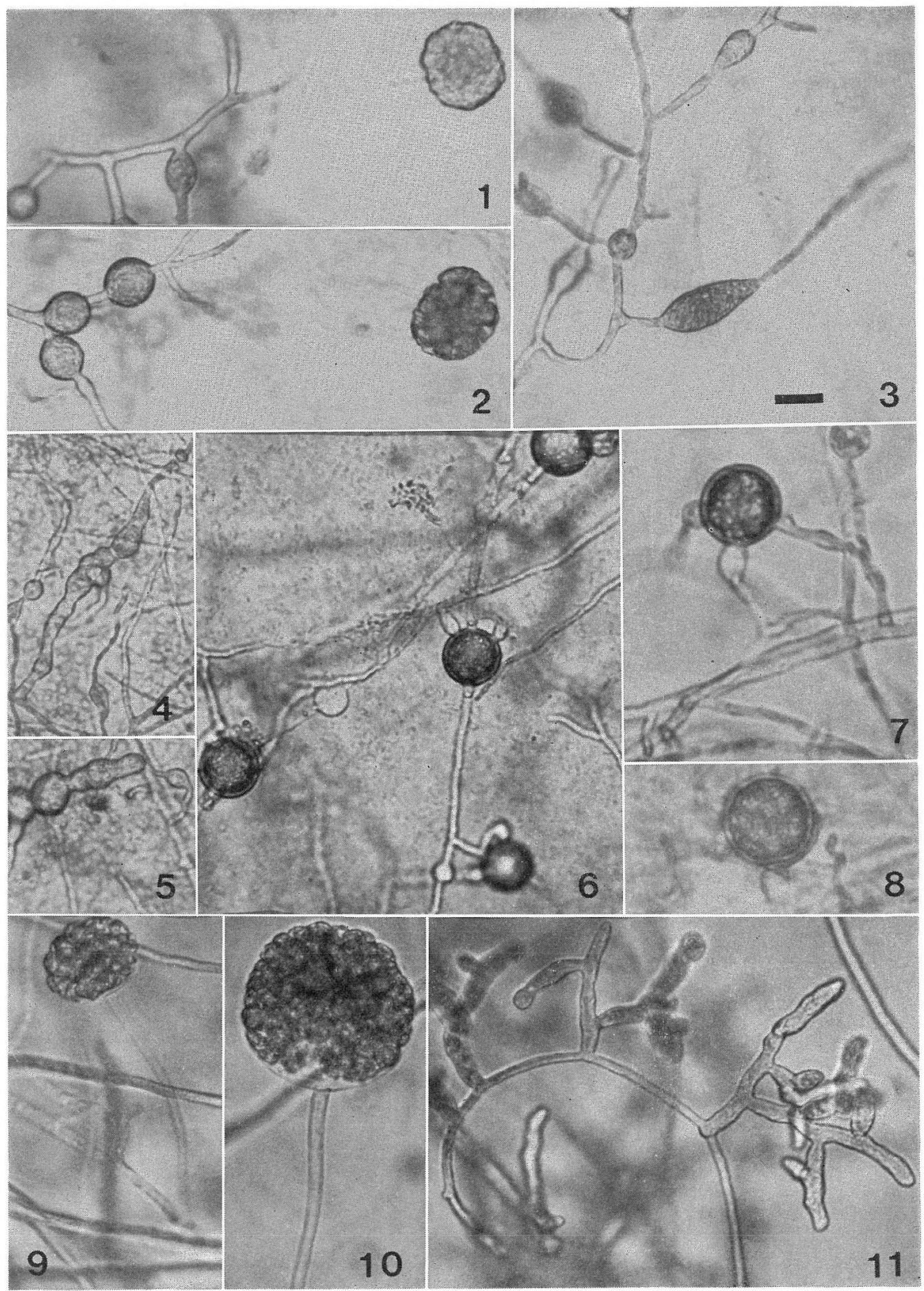

